\section{Microbiological findings in ready-to-eat and precooked food distributed in public catering halls in Cagliari Province, Italy}

\author{
Silvana Brignardello, ${ }^{1}$ Rosangela Sabiu, \\ Tiziana Tedde, ${ }^{2}$ Enrica Cocco, ${ }^{3}$ \\ Gabriella Pitzalis, ${ }^{4}$ Clara Meli, ${ }^{5}$ Maria \\ Paola Cogoni ${ }^{1}$
}

'Istituto Zooprofilattico Sperimentale

della Sardegna, Elmas (CA); ${ }^{2}$ Istituto

Zooprofilattico Sperimentale della

Sardegna, Sassari; ${ }^{3}$ Servizio Igiene degli

Alimenti e della Nutrizione, ASL 8, Cagliari; ${ }^{4}$ Servizio Igiene degli Alimenti e della Nutrizione, ASL 7, Carbonia (CI); ${ }^{5}$ Servizio Igiene degli Alimenti e della Nutrizione, ASL 6, Sanluri (VS), Italy

\section{Abstract}

During this research 159 samples of readyto-eat and precooked food were examined for the detection of Salmonella spp., Listeria monocytogenes, Escherichia coli, coagulasepositive staphylococci and Enterobacteriaceae, bacterial count. All samples were negative for Salmonella spp. and Listeria monocytogenes; although a low count of coagulase-positive staphylococci (no. 5 samples) and E. coli (no. 3) was found. We proceeded with the identification of the isolates on every sample with high, moderate and low-count of Enterobacteriaceae, to better understand the microbial ecology. The Enterobacteriaceae species most frequently detected were Pantoea spp. (29.62\%), Enterobacter cloacae (20.37\%) and Serratia liquefaciens (12.96\%); other different species of Enterobacteriaceae were detected with a $<10 \%$ presence. Since there are no legal regulations indicating tolerance or limit values or this kind of microorganisms related to the samples, it would be appropriate, in the presence of high count of Enterobacteriaceae or by type of ready-to-eat and precooked food, to proceed with the identification of every microorganism.

\section{Introduzione}

Nel periodo aprile 2012-aprile 2013 sono stati analizzati presso il Laboratorio di Microbiologia degli alimenti del Dipartimento di Cagliari dell'Istituto Zooprofilattico Sperimentale della Sardegna (IZS Sardegna)
159 campioni di piatti pronti e precotti, prelevati dal Servizo Igiene degli Alimenti e della Nutrizione (SIAN). I campioni sono stati suddivisi nelle seguenti tipologie alimentari: i) preparazioni gastronomiche precotte, ii) preparazioni gastronomiche cotte e spuntini, iii) insalate di mare, iv) dolci e dessert, v) insalate e ortaggi, vi) salse e sughi, vii) frutta e succhi, come riportato in un precedente lavoro (Nyenje et al., 2012). In tutti i campioni sono stati determinati i seguenti parametri microbiologici: Listeria monocytogenes (n. 118 campioni), Salmonella spp. (n. 131), Enterobacteriaceae (n. 130), Escherichia coli (n. 133), stafilococchi coagulasi positivi (n. 124), microrganismi mesofili aerobi (n. 10).

Lo scopo di questo lavoro è stato quello di acquisire informazioni per valutare la qualità microbiologica in campioni di piatti pronti e precotti, provenienti dalla ristorazione pubblica e collettiva distribuiti nel territorio della provincia di Cagliari e di approfondire lo studio di alcune specie batteriche presenti in elevata 0 moderata carica.

\section{Materiali e Metodi}

La ricerca di Listeria monocytogenes è stata effettuata secondo la Norma UNI EN ISO 11290-1:2005 (UNI, 2005); la ricerca di Salmonella spp. è stata effettuata secondo la Norma ISO 6579:2008 (ISO, 2008); la ricerca di Enterobacteriaceae è stata effettuata secondo la Norma ISO 21528-2:2004 (ISO, 2004); la ricerca di $E$. coli è stata effettuata secondo la Norma 16649-2:2001 (ISO, 2001); la ricerca di stafilococchi coagulasi positivi è stata effettuata secondo la norma UNI EN ISO 68882:2004 (UNI, 2004); la ricerca di mesofili aerobi è stata effettuata secondo la Norma ISO 4833:2003 (ISO, 2003). Per l'identificazione di specie sono stati utilizzati tests biochimici e gallerie miniaturizzate. In particolare, per l'identificazione delle Enterobacteriaceae è stata effettuata la colorazione di Gram e il test dell'ossidasi. Per l'identificazione di specie delle Enterobacteriaceae sono state utilizzate le gallerie API 20E (bioMérieux, Marcy l'Etoile, Francia). Per l'analisi statistica i valori UFC/g sono stati convertiti in $\log _{10}$; i valori numerici delle Enterobacteriaceae ottenuti nelle tipologie alimentari sottoposte ad analisi, sono stati comparati mediante analisi della varianza a una via (one-way ANOVA) seguito da un test di comparazione multipla di Tukey. L'analisi statistica è stata eseguita con SPSS versione 15.0 e sono stati considerati significativi i valori con $\mathrm{P}<0,05$.
Correspondence: Maria Paola Cogoni, Istituto Zooprofilattico Sperimentale della Sardegna, Via dell'acquedotto romano, 09132 Elmas, CA, Italy. Tel. +39.070.21914213 - Fax: +39.070.2135121.

E-mail: paola.cogoni@izs-sardegna.it

Key words: Ready-to-eat, Enterobacteriaceae, Microbiological quality.

Conflict of interests: the authors declare no potential conflict of interests.

Acknowledgments: the authors thank Dr. Cristian Pilo, research fellow at the Istituto Zooprofilattico Sperimentale della Sardegna (Sardinian institute to help prevent disease in domestic herds of animals) Cagliari, Italy, for his helpful collaboration.

Received for publication: 21 May 2013

Revision received: 13 January 2014.

Accepted for publication: 13 January 2014.

This work is licensed under a Creative Commons Attribution 3.0 License (by-nc 3.0).

CC Copyright S. Brignardello et al., 2014

Licensee PAGEPress, Italy

Italian Journal of Food Safety 2014; 3:1733

doi:10.4081/ijfs.2014.1733

\section{Risultati}

Tutti i campioni sono risultati negativi per Salmonella spp. (n. 131) e per Listeria monocytogenes (n. 118). In 5/124 e 3/133 campioni analizzati rispettivamente per stafilococchi coagulasi positivi e $E$. coli è stata riscontrata una carica accettabile secondo i parametri stabiliti dal Reg. (CE) n. 2073 del 2005 (Commissione Europea, 2005) e successive modifiche. Per meglio comprendere l'ecologia microbica nei campioni con una carica di Enterobacteriaceae $>10$ UFC/g (n. 54 campioni), si è proceduto alla identificazione degli isolati. Enterobacter spp. (31,5\%) è risultato il genere prevalente; le specie identificate sono risultate essere Enterobacter cloacae (20,37\%), Enterobacter agglomerans (5,55\%), altri (5,55\%) mentre, Pantoea spp. e Serratia spp. rappresentano rispettivamente il $29,6 \%$ e il $16,6 \%$ degli isolati (Tabella 1). Un valore elevato di Klebsiella pneumonie $\left(2,4 \times 10^{6}\right.$ ufc/g) è stato accertato in un campione di insalata di mare. L'analisi della varianza ha evidenziato una significatività statistica tra i valori delle Enterobacteriaceae $\left(\log _{10} \mathrm{UFC} / \mathrm{g}\right)$; il test di comparazione multipla (Tukey) ha dettagliato le tipologie di alimenti differenti tra loro $(\mathrm{P}<0,05)$ (Tabella 2). 
nelle piante per cui, dai dati raccolti, si può ritenere che tale microrganismo appartenga alla flora batterica naturale presente nelle verdure (Houa et al., 2013). La categoria insalate e ortaggi rappresenta la tipologia di prodotti alimentari maggiormente contaminati da Enterobacter cloacae. Tale dato è sovrapponibile ai risultati ottenuti da Falomir et al. (2010). Come riportato in letteratura (Jay et al., 2011), tale microrganismo è inserito tra gli otto batteri Gram-negativi che possiedono almeno un fattore di virulenza spesso associato a patogeni alimentari. I valori delle Enterobacteriaceae nei piatti precotti presentano un range elevato (Tabella 2), ma trattandosi di alimenti per i quali il trattamento termico riduce il valore della carica batterica, tale categoria di alimento non costituisce un rischio per il consumatore. Non è da sottovalutare tuttavia, la possibile presenza di alcune enterotossine termostabili prodotte da alcuni microrganismi come Enterobacter cloacae, Klebsiella pneumoniae (Jay et al., 2011) per i quali l'azione del calore risulta inefficace. Il piatto pronto insalata di mare è costituito da un prodotto base che comprende diverse specie ittiche cotte, verdure crude, ortaggi e spezie. Il prodotto base è presumibilmente privo di elevate cariche batteriche poiché sottoposto a cottura ma, l'aggiunta di verdure crude e spezie, può rappresentare una possibile fonte di contaminazione del prodotto finito. Le Enterobacteriaceae rappresentano un parametro inserito tra i criteri di igiene di processo [Reg. (CE) n. 2073 del 2005 e successive modifiche; Commissione Europea, 2005] con valori di riferimento associati. Nel caso in cui si effettui la ricerca di Enterobacteriaceae su matrici non inserite nel corpus legislativo, l'interpretazione del dato analitico è difficoltosa e la mancanza di valori di riferimento implica un confronto quasi esclusivo con la bibliografia scientifica.

\section{Conclusioni}

Sarebbe opportuno, in presenza di una carica medio alta e in assenza di valori di riferimento, procedere all'identificazione per la valutazione del rischio legato alla specie. A tutt'oggi, sulla base del risultato, l'autorità competente effettua le opportune verifiche sul processo di produzione e sulle misure

Tabella 1. Enterobacteriaceae: specie isolate da piatti pronti e precotti.

\begin{tabular}{lc} 
Specie & \% sul totale delle colonie identificate \\
Pantoea spp. & 29,62 \\
Enterobacter cloacae & 20,37 \\
\hline Serratia liquefaciens & 12,96 \\
Klebsiella oxytoca & 9,25 \\
\hline Enterobacter agglomerans & 5,55 \\
Hafnia alvei & 5,55 \\
\hline Enterobacter spp. (aerogenes, amnigenus, sakazakii) & 5,55 \\
Routella spp. & 5,55 \\
\hline Klebsiella pneumoniae & 3,70 \\
Serratia spp. (rubidaea, funticola) & 3,70 \\
\hline Citrobacter spp. & 3,70 \\
Altre specie & 11,11 \\
\hline
\end{tabular}

Tabella 2. Enterobacteriaceae in piatti pronti e precotti: statistiche descrittive e comparazione multipla (test Tukey).

\begin{tabular}{lccccccccc}
$\begin{array}{l}\text { Tipologia di } \\
\text { alimento }\end{array}$ & $\begin{array}{c}\text { Range } \\
\text { Enterobacteriaceace } \\
\left(\log _{10} \text { UFC/g) }\right.\end{array}$ & $\begin{array}{c}\text { MediatDS } \\
\text { Enterobacteriacear } \\
\left(\log _{10} \text { UFC/g) }\right.\end{array}$ & PPC & PC & IM & D & I0 & S & F \\
PPC & $<1,0-5,7$ & $2,18 \pm 2,35$ & - & 0,882 & 0,814 & 0,935 & 0,844 & 0,964 & 0,704 \\
PC & $<1,0-5,6$ & $1,41 \pm 0,92$ & 0,882 & - & 0,000 & 1,000 & 0,000 & 1,000 & 0,978 \\
\hline IM & $<1,0-6,6$ & $3,08 \pm 1,89$ & 0,814 & 0,000 & - & 0,003 & 1,000 & 0,005 & 0,002 \\
D & $<1,0-3,7$ & $1,44 \pm 0,83$ & 0,935 & 1,000 & 0,003 & - & 0,006 & 1,000 & 0,986 \\
\hline IO & $<1,0-5,0$ & $3,05 \pm 1,08$ & 0,844 & 0,000 & 1,000 & 0,006 & - & 0,010 & 0,004 \\
S & $<1,0-4,5$ & $1,53 \pm 1,13$ & 0,964 & 1,000 & 0,005 & 1,000 & 0,010 & - & 0,961 \\
\hline F & $<1,0-1,0$ & $1,00 \pm 0,00$ & 0,704 & 0,978 & 0,002 & 0,986 & 0,004 & 0,961 & - \\
\hline
\end{tabular}

DS, deviazione standard; PPC, preparazioni gastronomiche precotte; PC, preparazioni gastronomiche cotte e spuntini; IM, insalata di mare; D, dolci e dessert; IO, insalate e ortaggi; S, salse e sughi; F, frutta e succhi. 
messe in atto dall'operatore del settore alimentare. È auspicabile che, a livello regionale, si elabori un protocollo tecnico che preveda dei valori guida in relazione alla matrice e al risultato analitico, al fine di una migliore interpretazione e gestione del dato da parte degli enti interessati.

\section{Bibliografia}

Commissione Europea, 2005. Regolamento della Commissione del 15 novembre 2005 sui criteri microbiologici applicabili ai prodotti alimentari, 2073/2005/CE. In: Rivista Ufficiale, L 338/1, 22/12/2005.

Falomir MP, Gozalbo D, Rico H, 2010. Coliform bacteria in fresh vegetables: from cultivated lands to consumers. Available from: http://www.formatex.org/microbiology2/ch apters2.html

Houa Z, Finka RC, Radtkea C, Sadowsky MJ, Diez-Gonzalez F, 2013. Incidence of naturally internalized bacteria in lettuce leaves. Int J Food Microbiol 162:260-5.
ISO, 2001. Microbiology of food and animal feeding stuffs. Horizontal method for the enumeration of beta-glucuronidase-positive Escherichia coli. Part 2: colony-count technique at 44 degrees $\mathrm{C}$ using 5-bromo4-chloro-3-indolyl beta-D-glucuronide. Norma ISO 16649-2:2001. Organizzazione Internazionale per la Standardizzazione ed., Ginevra, Svizzera.

ISO, 2003. Microbiology of food and animal feeding stuffs. Horizontal method for the enumeration of microorganisms. Colonycount technique at 30 degrees C. Norma ISO 4833:2003. Organizzazione Internazionale per la Standardizzazione ed., Ginevra, Svizzera.

ISO, 2004. Microbiology of food and animal feeding stuffs. Horizontal methods for the detection and enumeration of Enterobacteriaceae. Part 2: colony-count method. Norma ISO 21528-2:2004. Organizzazione Internazionale per la Standardizzazione ed., Ginevra, Svizzera.

ISO, 2008. Microbiology of food and animal feeding stuffs. Horizontal method for the detection of Salmonella spp. Norma ISO 6579:2008. Organizzazione Internazionale per la Standardizzazione ed., Ginevra, Svizzera.

Jay JM, Loessner MJ, Golden DA, 2005. Modern food microbiology. Springer, Amsterdam, The Netherlands.

Nyenje ME, Odjadjare CE, Tanih NF, Green E, Ndip RN, 2012. Foodborne pathogens recovered from ready-to-eat foods from roadside cafeterias and retail outlets in Alice, Eastern Cape Province, South Africa: public health implications. Int J Environ Res 9:2608-19.

UNI, 2004. Norma UNI EN ISO 6888-2:2004. Microbiologia di alimenti e mangimi per animali. Metodo orizzontale per la conta di stafilococchi coagulasi-positivi (Staphylococcus aureus e altre specie). Tecnica che utilizza il terreno agar al plasma di coniglio e al fibrinogeno. Ente Italiano di Normazione ed., Milano, Italy.

UNI, 2005. Norma UNI EN ISO 11290-1:2005. Microbiologia di alimenti e mangimi per animali - Metodo orizzontale per la ricerca e la conta di Listeria monocytogenes. Parte 1: metodo per la ricerca. Ente Italiano di Normazione ed., Milano, Italy. 